\title{
Mulemba
}

Revista Angolana de Ciências Sociais

6 (12) | 2016

Sobre a sociedade e a cultura em Angola e alhures:

algumas reflexões de percepções sobre a realidade e múltiplas experiências

\section{A «raça» e o racismo: Algumas incidências sobre Angola}

«Race» and racism: Some reflections on Angola

\section{Arlindo Barbeitos}

\section{OpenEdition}

\section{Journals}

\section{Edição electrónica}

URL: http://journals.openedition.org/mulemba/603

DOI: $10.4000 /$ mulemba.603

ISSN: 2520-0305

\section{Editora}

Edições Pedago

Edição impressa

Data de publição: 1 novembro 2016

Paginação: 13-50

ISSN: 2182-6471

\section{Refêrencia eletrónica}

Arlindo Barbeitos, «A «raça» e o racismo: Algumas incidências sobre Angola», Mulemba [Online], 6

(12) | 2016, posto online no dia 30 setembro 2018, consultado o 26 janeiro 2021. URL: http://

journals.openedition.org/mulemba/603 ; DOI: https://doi.org/10.4000/mulemba.603 


\title{
L'intégration régionale en Afrique: Un processus déjà ancien... pour quels résultats?
}

\begin{abstract}
Résumé: Cet article est une analyse critique de l'histoire et de l'état actuel de l'intégration économique et politique du continent africain. Il constate que, malgré les efforts considérables consentis à cet égard, l'intégration régionale ne semble pas avoir encore produit les résultats escomptés. Il commence, en effet, par identifier les défis à relever par les programmes de l'intégration régionale en Afrique et ce, depuis son apparition avec l'idéal panafricain jusqu'ici. Aussi, il évoque les obstacles auxquels l'Afrique doit surmonter pour atteindre les objectifs visés par ses diverses Communautés économiques régionales (CER).En conclusion, l'article présente des perspectives d'espoir, car l'Afrique n'est pas vouée au désespoir tout comme les autres continents, notamment ceux du Tiers-monde, un terme en voie de disparition du vocabulaire des relations internationales depuis la fin de la guerre froide (1947-1989).
\end{abstract}

Mots clés: Afrique, coopération, dévelopement, intégration régionale, organisatrion internationale regionale, unité africaine.

En guise d'introduction, on peut dire qu'à lui seul, l'intitulé de cet article pose d'emblée la problématique essentielle de ce processus de coopération pour le développement entre pays africains suivant leurs régions respectives. Elles sont réparties selon les points cardinaux. Mais force est de constater que tant d'années après les indépendances et sa mise en place, les résultats probants se font attendre. Aborder la question de l'intégration régionale en Afrique nous ramène forcément au panafricanisme. D'où, nous verrons ce

* Enseignant d'Histoire au Département d'Histoire (DE) de la Faculté des Sciences Sociales (FCS) de l'Université Agostinho Neto (UAN). 
qu'il en est et ce qu'il a engendré en Afrique, dont le régionalisme en question, mieux connu sous le terme de l'intégration régionale.

\section{Le Panafricanisme: une question de l'unité des Peuples Noirs et des Africains}

Le panafricanisme ${ }^{1}$ est une doctrine tendant à unifier les Peuples d'Afrique en un seul. Ce fut la bataille de cheval de ses fervents partisans tant de la diaspora que d'Afrique. Né à la fin du xvirie siècle dans les Amériques, le panafricanisme était à ses débuts un mouvement socioculturel qui militait pour la solidarité et l'union de la diaspora africaine aux Caraïbes et États-Unis d'Amérique (EUA) pour retrouver, notamment au lendemain de l'indépendance des EUA (1776), ses droits civils, culturels et moraux face à l'injustice et à la souffrance subies sous la domination esclavagiste des Américains et Européens blancs.

Peu à peu, il se transforme en un mouvement politique qui se veut universel et rassembleur de tous les peuples noirs du monde. Il trouve naturellement à la fois sa source d'inspiration et son terrain d'expression en Afrique, alors totalement ou presque sous le joug colonial, à l'exception de l'Éthiopie et du Libéria. Bien qu'il soit l'une des plus anciennes civilisations et un État souverain depuis l'Antiquité, l'Égypte était à cette époque sous l'occupation ottomane dès 1517, puis sous le protectorat britannique (1914-1922). Non seulement, le panafricanisme appelle à la libération totale de l'Afrique de la domination coloniale mais aussi et, surtout, à son unité.

Pour ses fondateurs, ${ }^{2}$ il existe bien une personnalité africaine commune à tous les hommes, toutes les femmes de race noire.

1 On l'écrit soit en un seul mot (panafricanisme), soit en deux mots (pan-africanisme). Le «pan» vient du grec pán qui signifie «tout» et «africanisme» vient bien sûr d'Afrique et de ce qu'elle comporte ou engendre en matière de civilisation, de culture, de sociologie, de religion, etc.

2 Parmi eux, on peut citer les mieux connus de la diaspora africaine que sont Henry Sylvester William (Trinité et Tobago) considéré comme le précurseur; Edward Wilmot Blyden (universitaire et diplomate américano-libérien, dont les écrits sont considérés comme fondateurs du panafricanisme), William Eduard Burghardt Du Bois (EUA) qui en est le père; Marcus Mosiah Garvey (Jamaïque), l'apôtre du panafricanisme messianique; George Padmore (Trinité et Tobago), le théoricien du panafricanisme; et Jean Price-Mars (Haïti), le mentor du panafricanisme culturel. Côté africain, les plus engagés des militants panafricanistes sont: Hailé Sellasié (Ethiopie), l'hôte de l'ouA; Kwame Nkrumah (Ghana), 
Cette personnalité noire recèle des valeurs spécifiques de sagesse, d'intelligence et de sensibilité. Les peuples noirs qui sont les peuples les plus anciens de la terre (d'où le credo autant anthropologique que historique selon lequel «l'Afrique est le berceau de l'Humanité») sont voués à l'unité et à un avenir commun, ajoutent-ils. Le panafricanisme est, donc, par essence un mouvement d'idées et d'émotions, voire de mythes. C'est une vision culturelle, sociale et politique, une philosophie et une idéologie qui cherchent à unifier les Africains d'Afrique avec les membres de sa diaspora éparpillée à travers le monde en une communauté africaine globale, et qui appelle à l'unité politique de l'Afrique, faut-il encore le rappeler?

Tout en appelant les Africains à prendre conscience de leurs réalités multiples qu'il ne faut pas chercher à estomper, le panafricanisme les appelle aussi à ne pas se diluer ou s'abandonner, mais plutôt à s'affirmer. Il refuse toute idée d'assimilation, d'association et d'intégration dans le monde du dominateur blanc qui avait caractérisé le système colonial. Ainsi, il a soutenu avec force le mouvement de libération nationale en Afrique contre la domination coloniale. Son slogan était alors: "L'Afrique aux Africains». Mais il vise aussi à amener les Africains à participer à l'élaboration de la «civilisation universelle» ainsi qu'à la coopération économique, intellectuelle et politique entre les pays africains. Il demande que les richesses du continent soient utilisées pour le développement de ses peuples. Ainsi, il appelle à l'unification des marchés financiers et économiques et à un nouveau paysage politique du continent. Autrement, il a appelé à l'intégration régionale en question, appelé aussi le panafricanisme de l'intégration à côté de celui de la libération, lequel était principalement politique. Mais quand, pourquoi et comment ce mode de coopération est-il survenu sur le continent au point de supplanter l'idéal panafricain qui lui avait creusé le sillon?

l'idéologue et propagandiste; Benjamin Nnamdi Azikiwe (Nigeria); Ahmed Sékou Touré (Guinée Conakry); Jomo Kenyatta (Kenya), le doyen; Julius Nyerere (Tanzanie), le théoricien du socialisme africain; Patrice Émery Lumumba (RDC), le héros et martyr des indépendances africaines; Kenneth Kaunda (Zambie), l'homme au mouchoir blanc, signe de paix et de tolérance; Mouhamar el Kadhafi (Libye), le dernier partisan du panafricanisme maximaliste; et Peter Abrahams (Afrique du Sud), l'écrivain engagé. 


\section{De l'unité du continent à sa régionalisation}

Quand? Ce fut au cours de la vague des indépendances des années 1960 et la création consécutive de l'Organisation de l'Unité Africaine (OUA) en 1963. En fait, elle est l'institutionnalisation du panafricanisme sur le continent. Pourquoi? Parce que la toute nouvelle Afrique indépendante cherchait, via l'ouA, à tracer sa voie pour atteindre son double objectif: l'indépendance totale de tous les pays restés sous le joug colonial, la fin de l'apartheid comprise, et le développement socio-économique de ces mêmes pays au fur et à mesure qu'ils s'en libéraient. Comment? Partagé entre le panafricanisme des uns, selon le vieil adage «l'union fait la force», en ayant à l'esprit l'idée des «États-Unis d'Afrique» et le régionalisme des autres basé, lui aussi, sur le principe de «la solidarité africaine est une force». Ce dernier s'appuie sur le partage de l'Afrique en quatre, puis en cinq régions actuellement. C'est ainsi que le continent s'était vite retrouvé devant un dilemme, celui de l'embarras du choix politique pour mener à bien sa double action de libération politique et d'indépendance économique. De plus, il devait faire face au poids de la guerre froide (1947-1989) qui avait opposé ses pays entre modérés et progressistes suivant leurs orientations idéologiques respectives. Les premiers avaient pour alliés l'Europe Occidentale et les EUA tandis que les seconds pouvaient compter sur l'Europe de l'Est, l'ancienne Union Soviétique et la Chine communiste.

Entre les deux groupes, la bataille d'idées fut âpre. Au final, c'est le groupe des régionalistes dits des pragmatiques ou modérés qui l'avait emporté. C'est ainsi qu'on parle de régionalisme ou du panafricanisme d'intégration régionale aujourd'hui en Afrique. C'est un processus résultant d'une démarche volontaire de deux ou de plusieurs États différents en vue d'une mise en commun d'une partie ou de l'ensemble de leurs ressources. Ce processus a pour finalité l'émergence et le renforcement des relations techniques et économiques d'interdépendance structurelles à effets d'entraînement positifs. L'union qui en résulte correspond à la soumission de l'ensemble des partenaires et des espaces régionaux auxquels ils appartiennent à des politiques communes, dites aussi uniques.

Ses tenants dits du groupe de Monrovia (du 8 au 12 mai 1961) étaient emmenés par le président ivoirien Félix Houphouët-Boigny, alors que les panafricanistes du groupe de Casablanca (du 3 au 7 janvier 1961) étaient conduits par le président ghanéen Kwame 
Nkrumah. Ces derniers sont considérés comme les partisans du panafricanisme maximaliste pendant que les premiers ceux du panafricanisme minimaliste. Les deux courants ont leurs héritiers. Nonobstant ce désaveu, l'idéal panafricain n'a guère disparu au sein de l'Afrique. Au contraire, il continue de traverser les esprits des uns et des autres, tous bords confondus. Photos à l'appui, l'historienpanafricaniste Théophile Obenga indique quelques figures historiques du panafricanisme dans L'État fédéral d'Afrique noire: La seule issue (2012).

Le dernier à vouloir, en vain, le relancer en tant qu'héritier du panafricanisme maximaliste a été le président libyen Mouhammar Kadhafi, dont on connaît le sort, puisque tué lors du printemps arabe qui avait déferlé aussi sur la Libye (2011). Néanmoins, son implication personnelle et la contribution financière de son pays avaient permis la mutation de l'ous à l'Union Africaine (UA) en 2002. Principale organisation continentale, elle n'a cessé non plus de s'inspirer du panafricanisme dans la mise en place de ses grands projets politiques et économiques, comme ceux du NEPAD ${ }^{3}$ (2001), d'une Zone de libre-échange continentale (ZLEC) panafricaine à la date indicative de 2017, d'un marché commun africain à l'horizon 2023, d'un réseau de neuf autoroutes allant du Nord au Sud et d'Est en Ouest, ainsi que d'une ligne de chemin de fer traversant tous les pays au plus tard en 2063. Cet objectif ambitieux a un nom: c'est le fameux Agenda 2063, selon lequel «L'Afrique que nous voulons».

\section{Le symbole du régionalisme: Les CER}

Cet agenda et tous ses projets, entre autres, s'appuient sur les intégrations régionales, dont les grandes et principales organisations internationales régionales, appelées Communautés économiques régionales, soit lesdites CER (CAE, CEDEAO, CEEAC, SADC,

3 Le Nouveau Partenariat pour le développement de l'Afrique, acronyme de New Partnership for Africa's Development (NEPAD). C'est un programme de l'Union africaine (UA) qui vise à éradiquer la pauvreté, à placer les pays africains, individuellement et collectivement, sur la voie de la croissance et du développement durable, à renforcer la capacité de l'Afrique à participer activement à l'économie et à la politique mondiales et à accélérer l'autonomisation des femmes. 
UMA), ${ }^{4}$ sont considérées comme leurs piliers. Dans une approche comparative, nous les évoquons longuement dans notre thèse de doctorat (2011). ${ }^{5} \mathrm{Il}$ a fallu, cependant, attendre les années 1980 pour voir ces CER prendre leur timide envol à la suite du Plan d'action de Lagos. Il fut adopté lors d'un Sommet extraordinaire de l'ouA (1980). Elles ont toutes pour modèle l'Europe, de la Communauté Économique Européenne (CEE) à l'Union Européenne (UE). Et Annick Kamgang, dite KAM, une dessinatrice franco-camerounaise se demande dans un article paru le 24 juin 2016 sur le site du Jeune Afrique: "Et si le Brexit inspirait l'Afrique?», tant l'Afrique est habituée à s'inspirer des autres à bien des égards. Elle revient bien entendu sur le référendum britannique sur le maintien ou non du Royaume-Uni au sein de l'ue qui avait succédé à la cEE en 1992. Les sujets de Sa Majesté ont plutôt dit «Non» à 51,9 \% des voix contre 48,1\%, à l'issue du référendum du 23 juin 2016.

En conséquence, le pays est en principe sorti de l'ue, d'où le terme anglais Brexit qu'elle reprend aussi pour l'Afrique: «Afrixit». Elle s'interroge si l'Afrique pourrait faire autant, en lançant un débat sur sa souveraineté, notamment sur les questions monétaires et judiciaires pour se soustraire de l'emprise européenne. Il faut dire qu'en matière de monnaie, le Franc cFA utilisé dans dix-huit pays francophones est, par exemple, arrimé à l'Euro, alors que dans d'autres pays leurs monnaies sont indexées sur le dollar américain. Dans le domaine de la justice, la Cour pénale internationale (CPI) de La Haye ne traque que les dirigeants africains, selon nombre d'Africains. Sur dix procès en cours à la cPI, neuf concernent des pays africains. C'est ainsi que des pays tels que l'Afrique du Sud, ${ }^{\mathbf{6}}$

4 CAE: Communauté d'Afrique de l'Est; CEDEAO: Communauté Économique de Développement des États de l'Afrique de l'Ouest; CEEAC: Communauté Économique des États de l'Afrique Centrale; SADC: Southern African Development Community (Communauté de Développement de l'Afrique Australe); uMA: Union du Maghreb Arabe.

5 L'intégration régionale en Afrique australe: De la Ligne de Front à la $S A D C$, Thèse de Doctorat en Histoire contemporaine, Université Paris I - Sorbonne, 2010, 969p., soutenue le 2 décembre 2010, elle a été publiée par l'Atelier national de reproduction des thèses (ANRT) en 2011.

6 Selon le ministre sud-africain de la Justice, la décision de quitter la cPI n'était pas facile à prendre. Elle fait suite à une vive controverse entre l'Afrique du Sud et la CPI qui lui reproche de ne pas avoir arrêté le Président soudanais El Bachir en visite dans le pays pour un sommet de l'uA en juin 2015, alors qu'il était sous mandat d'arrêt de la cPI. 
le Burundi $^{7}$ et la Gambie se sont retirés de la CPI. D'autres, comme l'Ouganda, envisagent de leur emboîter le pas. Selon eux, la CPI s'acharne sur l'Afrique. Et pourtant, le continent vient de donner un exemple en la matière avec le procès historique de l'ancien président tchadien Hissène Habré. Il a été jugé et condamné à la perpétuité en mai 2016 par un tribunal spécial au Sénégal, au nom de l'Union Africaine, pour crimes contre l'humanité, crimes de guerre et torture commis sous son régime (1982-1990). Ayant refusé de reconnaître l'autorité de ce tribunal, il a fait appel de cette condamnation. Le procès en appel a débuté le 9 janvier 2017, pendant que nous finalisions ces lignes. Son verdict est attendu pour le 27 avril 2017, date à laquelle l'ancien chef d'État tchadien sera fixé sur son sort.

En revanche, Cécile Kyenge, originaire de la RDC, députée européenne et ancienne ministre italienne de l'Intégration, est à la fois partagée et critique de ce qu'elle appelle «le bal des abandons» dans un article du 7 novembre 2016 publié sur le même site du Jeune Afrique sous le titre «Du Brexit de l'UE à l'Afrexit de la CPI». Elle pense que le Royaume-Uni a plutôt envoyé un signal choc aux détracteurs aussi bien de l'intégration régionale que de la communauté internationale, même si c'est la voix du peuple qui a parlé par référendum alors que les pays africains abandonnent la cPI par simple «annonce gouvernementale». Elle y voit des mauvaises pratiques politiques et, surtout, le souci des dirigeants africains de vouloir sauvegarder leur immunité, pendant qu'ils tripotent les constitutions pour leurs intérêts personnels et ne garantissent vraiment pas les droits des peuples. Comme d'autres, elle n'est pas tendre non plus avec la CPI qu'elle trouve paternaliste en pratiquant une politique néocoloniale à l'endroit des Africains et, à un degré moindre, des Européens du Centre et de l'Est. Pour sa part, Francis Dako (2016) ${ }^{\mathbf{8}}$ estime que ces abandons de la CPI ne sont ni plus ni moins «des mouvements d'humeur sans base légale». Il est de ceux qui pensent que les dirigeants de ces pays sortants le font pour se soustraire tout simplement

7 Son Parlement a voté, le 13 octobre 2016, le retrait du pays de la cPI mais sans lui en avoir formalisé officiellement.

8 Voir son article «Les retraits de la cPI sont des mouvements d'humeur sans base légale» du 3 novembre 2016 sur <http://www.jeuneafrique.com/371141/politique>, consulté le 9 novembre 2016. On peut comprendre son avis puisqu'il est avocat au Barreau du Bénin, directeur du Cabinet Dako \& Co et ancien Coordinateur Afrique de la Coalition pour la CPI. 
du droit et de la justice internationaux. Mais que dire des EuA, de la Chine qui n'ont jamais signé le Statut de Rome sur la CPI et de la Russie qui vient d'en retirer sa signature?

Pour revenir à A. Kamgang, les choses ne sont pas si simples que cela tant l'ue est de loin l'important partenaire et bailleur de fonds historique des pays africains avec 129 milliards de dollars d'échanges commerciaux en 2010. Elle vient avant les États-Unis qui n'occupent que la troisième position dans les échanges avec l'Afrique (95 milliards de dollars en 2011), et après la Chine dont le volume des échanges avec l'Afrique a atteint 166,3 milliards de dollars en 2011.

En plus les responsables des banques africaines concernés par la remise en cause du Franc CFA, qui fait couler beaucoup d'encre, ne sont pas favorables à la rupture de cette monnaie avec l'Euro. Pour eux, être sous la bannière de la monnaie unique européenne est une assurance et une garantie face aux fluctuations et turbulences tant monétaires que financières qui secouent des temps en temps le monde. Et Sylvie Guillaumont ${ }^{9}$ d'affirmer que: «Le CFA n'est pas si mauvais». Par ailleurs, l'ue est, en effet, leur pourvoyeuse d'aide aussi. À titre d'exemple, prenons le cas de l'Afrique de l'Ouest, donc de la CEDEAO, qui apparaît comme une région privilégiée par l'UE. Cette région aurait bénéficié d'une enveloppe financière de coopération régionale croissante au fil des conventions: Lomé I (94,9 millions d'Écu); ${ }^{10}$ Lomé II (141 millions d'Écu); Lomé III (213 millions d'Écu); Lomé IV-1 (228 millions d'Écu).

Cette tendance à la hausse ne saurait occulter le fait que la part de l'Afrique de l'Ouest, dans la programmation des aides régionales européennes, a régulièrement décru passant de 42,5\% au cours de la convention de Lomé I à 30,1 \% sous Lomé II, 29,3\% sous Lomé III et 29,2 \% sous Lomé IV-1, selon Bernard Conte $(1997)^{11}$ du CEAN

${ }^{9}$ Chercheuse au Centre d'études et de recherches sur le développement international (CERDI), elle a été la grande invitée de l'Économie RFI - Jeune Afrique. Voir l'article de Frédéric Maury, «Sylvie Guillaumont: "Le CFA n'est pas si mauvais"», du 2 décembre 2016 sur <http://www.jeuneafrique.com/379249/economie/ sylviane-guillaumont-cfa-nestmauvais/?utm_source=Newsletter_JA_Eco\&utm_ medium=Email\&utm_campaign=Newsletter_JA_Eco_02_12_16>, lu le 3 décembre 2016.

10 Entre l'Euro et l'Écu, c'est la parité: un euro pour un écu. Ce dernier est, depuis 1979, l'acronyme de l'unité monétaire européenne (European Currency Unit) à laquelle l'euro a succédé en 1999, mais mis en circulation en 2002.

11 Lire son article «L'aide de l'Union européenne dans le domaine de l'intégration 
- Centre d'étude d'Afrique noire de l'Université Montesquieu Bordeaux Iv. Entre coupes et gels, les enveloppes allouées à l'aide publique au développement (APD) par les pays riches sont en baisse depuis les années 1990. En Espagne, par exemple, l'APD accordée au Maroc est passée de 90 à 15 millions d'euro entre 2010 et 2012, soit une réduction de $75 \%$. La crise économique et financière dont souffre l'Espagne, mais l'ue aussi, depuis 2008 est passée par là. Le pays est plongé dans la récession et soumis à une cure d'austérité historique sur fond de malaise social, même si «le pire de la crise est derrière nous» avait déclaré le ministre espagnol de l’Économie et de la Compétitivité, Luis de Guindos Jurado, en juillet 2014.

\section{Les problèmes d'intégration régionale en Afrique}

La dépendance technique, matérielle et financière de ces CER à l'aide étrangère constitue l'un de leurs points faibles parmi d'autres. Malgré la mise en place des groupes (ou forces) d'interposition, les CER ont du mal à préserver la paix dans les États membres menacés ou touchés par des conflits armés sans l'intervention étrangère comme au Mali, alors qu'il y a l'Ecomog dans la CEDEAO, ou encore en République Centrafricaine (RCA). Il en est de même dans d'autres secteurs comme celui de la santé publique face à des épidémies telles que Ebola qui aurait fait, entre les mois de mars et de décembre 2014, plus de 8000 victimes dans la CEDEAO (Guinée, Liberia, Sierra Leone) selon le bilan de l'oms (début 2015), ainsi que le sIDA dans la SADC en Afrique australe. Elle est considérée comme la région ayant le taux le plus élevé de prévalence de cette pandémie au monde, avec plus de 13 millions de personnes infectées en 2014. Autant dire que les CER sont encore bien loin du compte.

Il faut dire qu'elles n'offrent pas encore, sur le plan structurel, un cadre propice à l'activité productive, à la croissance soutenue et à l'emploi (des jeunes en particulier): source de richesse et de développement. Les égoïsmes nationaux et la lourdeur administrative y sont pour quelque chose. De surcroît, ces CER souffrent de la faiblesse des institutions démocratiques, de l’insuffisance des résultats

régionale: l'exemple de l'Afrique de l'Ouest», Cahiers du GEMDEV (Paris), n. ${ }^{\circ}$ 25, juin 1997, pp. 155-165. 
économiques (malgré quelques petites embellies par ci par là) et de l'absence de droits politiques, sociaux et civils. Elles présentent aussi un déficit politico-juridique qui se traduit par le non respect des règles et directives communautaires.

Autre lacune, et non la moindre, les populations pour lesquelles elles sont censées agir afin de leur faciliter la vie, ne se sentent pas concernées par leur action. Et pour cause, elles ne savent ni qui les dirigent, ni comment se transposent et s'appliquent leurs directives à l'échelle nationale. Donc, il y a là aussi un manque d'informations à l'égard des communautés nationales, malgré la présence des commissions nationales des CER dans les pays membres. S'y ajoute le secteur informel dont le rôle est plutôt néfaste pour les États et, donc, pour la région qui voient des rentrées fiscales leur échapper. De l'argent frais qui aurait pu être injecté dans les différents projets de développement régionaux. Par contre, il permet à de nombreuses familles à tirer leur épingle du jeu au point de passer d'un groupe social modeste à celui de la classe moyenne.

Selon les régions, on peut, toutefois, mettre sur leur compte un certain nombre de points positifs tels que la relative «bonne gouvernance» et l'ancrage de la démocratie dans la SADC (6 pays sur 15 et 4 anciens présidents lauréats du Prix Mo Ibrahim pour le leadership d'excellence en Afrique); la relative bonne santé économique dans la CEDEAO (emmenée par le Nigeria, le pays le plus peuplé d'Afrique - 182 millions d'habitants - qui, de 2014 à 2016 avait été aussi la première puissance économique du continent avec un PIB estimé à 568,5\$ milliards devant l'Afrique du Sud avec 384,3 milliards de dollars, ayant celle-ci récupéré son rang à la suite de la crise nigériane actuelle). La CEDEAO peut compter aussi sur le «retour en force» de la Côte d'Ivoire à laquelle l'agence de notation Fitch vient d'attribuer, pour la première fois, une note souveraine $B^{12}$ et sur le Ghana qui en détenait déjà. On peut dire autant de l'umA en Afrique du Nord avec des pays pris individuellement. Car l'uma en tant que CER est paralysée par la querelle algéro-marocaine sur l'inextricable problème saharaoui. Au niveau des pays, on constate la «montée en puissance» du Maroc métamorphosé ${ }^{\mathbf{3}}$ en dépit des

\footnotetext{
12 Cette note est toutefois inférieure d'un cran à celle (B1) accordée au pays par Moody's, l'autre agence de notation.

$13 \mathrm{Au}$ regard de cette embellie, le roi est optimiste de pouvoir faire entrer son pays au sein du groupe restreint des «pays émergents» dans les prochaines années. Lire
} 
disparités sociales criantes et l'expertise de la Tunisie, outre le potentiel économique algérien et libyen fondé sur le pétrole.

Petit bémol, le printemps arabe de 2011 a laissé des traces douloureuses, à l'exception de l'Algérie et du Maroc, où le vent de liberté a peu soufflé et qui s'appuient sur des pouvoirs publics forts. En revanche, la Libye peine à se faire une santé politique, ce qui constitue une source d'énormes inquiétudes économiques alimentée par les milices armées qui plongent le pays dans le chaos, pendant que la Tunisie est en quête d'un nouvel élan après celui d'une transition postrévolutionnaire complexe et agitée. Elle doit en plus gérer l'afflux massif de Libyens fuyant leur pays en pleine délicatesse.

À propos de la Libye qui est à feu et à sang, certains observateurs ${ }^{14}$ s'interrogent sur la pertinence de la campagne de bombardements aériens des Occidentaux (Américains, Anglais et Français) qui avait permis aux rebelles de prendre le dessus sur M. Kadhafi, pendant que d'autres, comme Sarah Diffalah (2014), ${ }^{15}$ se demandent si fallait-il vraiment le renverser ? C'est bien connu: «le sang appelle le sang». Là où l'on a appliqué cette méthode, l'État s'est délabré comme en Irak avec l'exécution par pendaison de Saddam Hussein le 30 décembre 2006 à Bagdad. Et les deux pays seraient progressivement devenus une sorte de hubs, voire de jihadistans, où les groupes islamistes armés viennent s'approvisionner en armes et se réorganiser. Au moment où, nous écrivons ces lignes, il semble que ces mouvements islamistes perdent du terrain face à l'offensive des forces gouvernementales.

Revenant aux CER, la CAE (Communauté d'Afrique de l'Est, plus connue sous son acronyme anglais d'EAC - East African Community - littéralement Communauté est-africaine, soit la CEA) se distingue par ses avancées dans la mise en place d'un marché commun (2010), d'une union douanière (2014) et bientôt d'une union monétaire, préalable à la mise en œuvre d'une monnaie unique: le Shilling est-africain dont le lancement initialement prévu en 2012 aurait été reporté autour de 2016. Grâce au marché commun, les cinq pays de la CEA (Burundi, Kenya, Ouganda, Rwanda, Tanzanie) affichent un dynamisme économique homogène avec une croissance

«Mohammed vi: le Maroc peut intégrer "le concert des pays émergents" » <http:// www.jeuneafrique.com>, du 28 août 2014.

14 Lire Le Monde (Paris), du 30 juillet 2014.

15 Du Nouvel Observateur. Voir son article du 5 août 2014 sur le site de ce magazine. 
régionale estimée à 6,1 \% en 2013. Son succès potentiel attirerait d'autres pays voisins à l'adhésion.

À en croire les spécialistes de la région, à commencer par ceux de l'Observatoire de l'intégration régionale en Afrique, le Soudan du Sud serait le prochain candidat à intégrer la CEA dans le court terme, pendant que la Tanzanie (pays-siège) milite, elle aussi, pour l'élargissement de la CEA à des pays tels le Malawi, la RDC et la Zambie. D'aucuns assurent que la CEA joue à fond la carte de l'intégration régionale pour le développement socio-économique de ses pays membres. À terme, elle aurait pour ultime objectif: la Fédération d'Afrique de l'Est. Une union politique qui agrégerait la totalité des pays membres en un État fédéral souverain (à l'instar de l'exemple américain) et dont la date proposée d'instauration avait été fixée à 2015. On peut penser que la crise burundaise, occasionnée depuis l'annonce de la candidature fin avril 2015 à un troisième mandat du président Pierre Nkurunziza à sa réélection contestée en juillet cette même année, aurait perturbé ce calendrier, comme celui de la mise en circulation de la monnaie unique régionale ci-dessous mentionnée.

En revanche, empêtrée dans des conflits de différents ordres depuis des années, la CEEAC en Afrique centrale, dont le Burundi est aussi membre, serait le maillon faible de ces CER. Et pourtant elle regorge d'énormes ressources naturelles, dont les minerais et, surtout, la forêt équatoriale du Bassin du Congo, le deuxième massif forestier tropical au monde après celui de l'Amazonie en Amérique latine. Ainsi, elle bénéficie d'un climat propice à l'agriculture, désignée comme la mamelle nourricière de l'Afrique. Elle a donc tout ou presque pour réussir son intégration régionale avec une jeunesse qui ne demande qu'à travailler, ni t'étais l'instabilité politique récurrente. Toutefois, l'espoir est permis tant qu'il fait vivre.

\section{Une lueur d'espoir?}

On peut le penser... Car la progression encourageante de certaines CER donne, au final, une lueur d'espoir en vue des lendemains africains prometteurs au point qu'on parle même de l'afro-capitalisme en devenir. Et les Bâtiment et Travaux Publics (втP) en donnent l'exemple avec le concours chinois qui concerne presque tous les pays africains. Comme l'a dit l'homme politique français Martin Na- 
daud (1815-1898): «Quand le bâtiment va, tout va». Pour preuve, la valeur totale des projets de construction en Afrique a accru de 46,2 \% en 2014 en atteignant la somme de 325 milliards de dollars américains. En cette même année, 257 projets d'infrastructures ont été recensés sur le continent, dont 119 en Afrique australe de la SADC considérée comme la première région d'investissements en la matière, suivie par l'Afrique occidentale de la CEDEAO avec 66 projets et par l'Afrique orientale de la CEA avec 51 projets. Quarante pourcents de ces projets concernent le secteur des transports, trente-neuf le secteur d'énergie alors que cinq pourcents touchent les secteurs du pétrole et du gaz, selon Deloitte ${ }^{\mathbf{1 6}}$ African Construction Trends Report (2014).

Dans cette dynamique, le secteur immobilier n'est pas en reste et connaît une croissance sans précédent. À en croire la revue américaine Engineering News-Record (2010), le secteur a vu ses ventes augmenter de 6,7\% pour atteindre la somme de 46 milliards de dollars américains, alors qu'ailleurs (EUA, UE, Moyen Orient) elles étaient nettement en baisse. En effet, beaucoup de pays africains ont lancé de grands projets de construction d'habitation, y compris des logements sociaux à la suite de l'explosion urbaine de ces dernières années. Le groupe immobilier marocain Alliances en tire la part du lion. Il a signé un projet de 40 ooo logements avec le Sénégal, de 14 ooo avec la Côte d'Ivoire et de 4 ooo avec le Congo-Brazzaville. À cela s'ajoute le boom spectaculaire du BTP chinois sur le continent. Depuis plus de dix ans, les Chinois multiplient les contrats en Afrique, sur le modèle controversé de «matières premières contre infrastructures», dit-on.

C'est ainsi que l'offensive chinoise dans le BTP africain ne faiblit pas. Qu'il s'agisse de la construction d'infrastructures énergétiques (gazoducs, raffineries, barrages), de transport (routes, chemins de fer, ports et aéroports) ou même de l'immobilier, les entreprises chinoises sont devenues incontournables sur le continent africain. Selon la Banque mondiale, elles détenaient près de $40 \%$ de part de marché d'un secteur estimé à près de 20 milliards d'euro en 2009 (dernier chiffre disponible). Au total, la Chine fait état de près de 500 projets sur lesquels se positionnent ses groupes en Afrique.

16 Un des grands cabinets d'audits et de conseils au monde créé en 1845 à Londres (Royaume-Uni), dont le siège social est à New York (EUA). 
À cet égard, l'Association internationale de constructeurs chinois (Chinca) joue un rôle essentiel. Cet organisme parapublic dispose d'une structure de veille des marchés forte de cinq cents personnes. A chaque appel d'offres, non seulement l'information est largement diffusée mais des délégations de professionnels sont immédiatement dépêchées sur place pour conseiller les autorités locales. Dans le même temps, le gouvernement chinois octroie des aides significatives aux entreprises qui veulent y contribuer. Et le résultat est là! En Algérie, dix-huit sociétés chinoises sont engagées dans des grands travaux, représentant $32 \%$ des gros contrats. Et même dans le pré-carré britannique (Ghana, Nigeria, Soudan), français (Bénin, Gabon) et portugais (Angola), les chantiers chinois pullulent, faisant sortir de terre des villes entières.

Désormais, le втр chinois rayonne sur l'Afrique au point de susciter des envies autant chez les Occidents, partenaires traditionnels des Africains, que chez leurs nouveaux partenaires comme la Turquie. Même le Japon veut désormais être en première ligne pour soutenir le développement de l'Afrique. Au terme de la sixième édition de la Conférence de Tokyo pour le développement en Afrique (TICAD VI), qui s'est tenu du 27 au 29 août 2016 à Nairobi (Kenya), les Japonais ont annoncé la création d'un Forum économique public-privé nippo-africain, avec à la clé un soutien financier de l'ordre de 10 milliards de dollars d'ici à 2018, année de la prochaine Ticad VII.

Il faut dire qu'en peu de temps, les Chinois réalisent ce que les Occidentaux n'ont pu faire sous leurs empires coloniaux pendant un siècle, pour reprendre la période avancée par les historiennes françaises Hélène d'Almeida-Topor et Monique Lakroum (1994), encore qu'à leur époque les performances des prouesses technologiques, dont bénéficient actuellement les Chinois, n'étaient pas ce qu'elles sont aujourd'hui. Et pourtant, les Occidentaux en sont les inventeurs dans la plupart des cas. C'est peut-être une question de volonté politique qui fait la différence dans cette aide au développement de l'Afrique. Elle est canalisée par un instrument de taille: le Forum sur la coopération sino-africaine.

Organisé tous les trois ans depuis 2000, il est une tribune de discussion pour une plus grande coopération économique entre la Chine et l'Afrique. Aussi, il est devenu un évènement clef pour les économies et diplomaties africaines et chinoises au cours du quel d'importants accords d'entraide et des contrats faramineux sont 
annoncés. Néanmoins, la cartographie des investissements chinois dans le monde place l'Afrique en avant-dernière position, avec 27,0 milliards de dollars, devant l'Océanie avec 22,7 milliards de dollars, comme l'indique le tableau ci-après:

Tableau n. ${ }^{\circ}$ 1. Les investissements chinois dans le monde (2014)

\begin{tabular}{c|c|c|c|c|c|c|c}
\hline Continent & Afrique & $\begin{array}{c}\text { Amérique } \\
\text { du Nord }\end{array}$ & $\begin{array}{c}\text { Amérique } \\
\text { du Sud }\end{array}$ & Asie & Europe & Océanie & Total \\
\hline $\begin{array}{c}\text { Stock en } \\
\text { milliards } \\
\text { de dollars } \\
\text { américains }\end{array}$ & 27,0 & 38,1 & 97,2 & 505,7 & 57,9 & 22,7 & 748,6 \\
\hline
\end{tabular}

Source: L'auteur à partir de Thierry Pairault (2016)

Sur une période plus ou moins longue d'une dizaine d'années, une autre étude sur l’investissement chinois à l'étranger, menée par les infographes du Courrier International et publiée sur le site de ce journal français, situe l'Afrique, notamment subsaharienne, au même rang presque (voir tableau ci-dessous).

Tableau n. ${ }^{\circ}$ 2. L'investissement chinois à l'étranger (2005-2016)

\begin{tabular}{|c|c|c|c|c|c|}
\hline \multirow{2}{*}{$\begin{array}{c}\text { Région } \\
\text { géographique }\end{array}$} & \multirow{2}{*}{$\begin{array}{c}\text { Afrique } \\
\text { Subsaharienne }\end{array}$} & \multicolumn{2}{|c|}{ Amérique } & \multicolumn{2}{|c|}{ Asie } \\
\hline & & $\begin{array}{c}\text { Amérique } \\
\text { du Nord }\end{array}$ & $\begin{array}{l}\text { Amérique } \\
\text { du Sud }\end{array}$ & $\begin{array}{c}\text { Asie } \\
\text { Occidentale }\end{array}$ & $\begin{array}{c}\text { Asie } \\
\text { Orientale }\end{array}$ \\
\hline $\begin{array}{l}\text { Investissement } \\
\text { en milliards } \\
\text { de dollars }\end{array}$ & 241,8 & 194,7 & 125,1 & 206,6 & 170,6 \\
\hline \multicolumn{6}{|l|}{$\nabla$} \\
\hline $\begin{array}{c}\text { Région } \\
\text { géographique }\end{array}$ & $\begin{array}{l}\text { Australie } \\
\text { (Océanie) }\end{array}$ & Europe & \multicolumn{2}{|c|}{$\begin{array}{l}\text { Moyen-Orient et } \\
\text { Afrique du Nord }\end{array}$} & Total \\
\hline $\begin{array}{l}\text { Investissement } \\
\text { en milliards } \\
\text { de dollars }\end{array}$ & 86,6 & 203,0 & \multicolumn{2}{|c|}{126,6} & 1354,9 \\
\hline
\end{tabular}

Source: L'auteur à partir de Cathy Doutey et Thierry Gauthé (2016)

Les investissements chinois se sont considérablement diversifiés même s'ils ne représentent que 3\% en 2014 en Afrique (cf. Tableau 1). Ici, ils intègrent principalement les secteurs de construction, de l'énergie et des métaux. La Chine étant devenue le 
premier partenaire commercial de l'Afrique, devant l'Europe et les États-Unis, les échanges sino-africains augmentent très vite, disions-nous. ${ }^{17}$ C'est ainsi que ce forum est de plus en plus considéré comme plus important que les autres.

Il s'agit notamment par rapport au sommet Europe-Afrique lancé aussi en 2000 au Caire (Égypte), puis débaptisé de «dialogue $U E$-Afrique» au sommet de Lisbonne en 2007 ainsi que du sommet États-Unis-Afrique (2014) qualifié, à juste titre, par certains observateurs de "permettre aux États-Unis de rattraper le temps perdu en Afrique face aux économies émergentes» comme la Chine et l'Inde. Et cette volonté chinoise ne laisse personne indifférente, y compris les Occidentaux qui cherchent à reprendre les choses en main dans bien de secteurs, dont le втр.

En effet, la population urbaine en Afrique atteindrait $60 \%$ en 2050 contre moins de $40 \%$ actuellement. C'est ainsi qu'il est urgent d'investir dans la construction des infrastructures et des logements, estime l'un-Habitat (le PNUEH -Programme des Nations unies pour les établissements humains) ${ }^{\mathbf{1 8}}$ dans son rapport sur l'état final des villes africaines publié en 2010. Même son de cloche du côté de Simon Walley (2014), coordinateur du programme immobilier à la Banque mondiale, qui pense que «Nous devons construire un million d'appartements et de maisons additionnelles chaque année pour répondre à la pression urbaine actuelle» pour ensuite ajouter que «L'Afrique sera capable de résoudre la crise d'habitat dans les quinze années à venir».

\section{Pour conclure}

Ces avancées et cet afro-optimisme sont les bienvenus tant qu'il était temps... À condition cependant que les mentalités tirent davantage vers le haut et que les pratiques changent aussi dans le sens d'une gouvernance digne de ce nom pour démentir l'ancien président de l'Assemblée nationale portugaise et les afro-pessimistes. En marge du Forum sur la citoyenneté et l’intégration régionale tenu

\footnotetext{
17 Voir les données d'échanges commerciaux à la page 3.

18 Il vient d'adopter un «nouvel agenda urbain» au terme de sa 3e Conférence sur le logement et le développement urbain durable, tenue à Quito (Equateur), du 17 au 20 octobre 2016. La première (Habitat I) eut lieu à Vancouver (Canada) du 31 mai au 11 juin 1976 et la deuxième (Habitat II) à Istanbul (Turquie) en 1996.
} 
début mai 2014 à Praia (Cap Vert), M. António Almeida Santos avait déclaré que «As ditaduras em África nunca acabarão, embora haja a tendência para que o seu número diminua» («les dictatures ne prendront guère fin en Afrique, bien que la tendance soit à la baisse»). Si ce n'est pas un défi lancé aux Africains, qu'est-ce que c'est?

\section{Références bibliographiques}

АвI-SAAB Georges

1980, Le concept d'organisation internationale. Paris, UNESCO, 292p.

AlmeIDA Érica Reis de

2007, O Pan-Africanismo: da OUA à UA. Trabalho para obtenção do diploma de Bacharel em Geografia. Niterói, RJ, Universidade Federal Fluminense.

Asante S.K.B. e Chanaiwa David

2010, «O Pan-Africanismo e a integração regional» in Ali Mazrui e Christophe Wondji (eds.), História geral da África, vII: África desde 1935. Brasília, unESCO - Representação do Brasil; Ministério da Educação e Universidade Federal de São Carlos, pp. 873-896.

BACH Daniel C.

1995, «Les dynamiques paradoxales de l'intégration en Afrique subsaharienne: le mythe du hors-jeu», Revue française de science politique (Paris), vol. 45, n. ${ }^{\circ}$ 6, pp. 1023-1038.

BORELLA François

1966, «Le régionalisme africain en crise (1965-1966)», Annuaire français de droit International, vol. 12, n. ${ }^{\circ}$ 1, pp 756-783.

1963, "Le régionalisme africain et l'Organisation de l'Unité Africaine», Annuaire français de droit International, vol. $9, \mathrm{n}^{\circ} \mathrm{1}, \mathrm{pp}$. 838-865.

1961, «Les regroupements d'États dans l'Afrique indépendante», Annuaire français de droit International, vol. 7, n. $^{\circ}{ }^{1}$, pp. 787$-807$.

BOUKARI-YABARA Amzat

2014, Africa Unite! Une histoire du Panafricanisme. Paris, La Découverte, $318 \mathrm{p}$.

Boutros-GHaLI Boutros

1969, L'Organisation de l'Unité africaine. Paris, Armand Colin, $196 \mathrm{p}$. 
1949, Contribution à l'étude des ententes régionales. Paris, A. Pedone, 247p.

CEA-UA

2008, État de l'intégration régionale en Afrique III. Vers l'intégration monétaire et financière en Afrique. Addis-Abéba, Commission économique des Nations unies pour l'Afrique, 306p.

2006, État de l'intégration régionale en Afrique II. Rationalisation des communautés économiques régionales. Addis-Abéba, Commission économique des Nations unies pour l'Afrique, $165 \mathrm{p}$. 2004, État de l'intégration régionale en Afrique I. Une étude de la CEA. Addis-Abéba, Commission économique des Nations unies pour l'Afrique, 304p.

Constantin François

1972, «L'intégration régionale en Afrique noire. Esquisse sur dix années de recherche», Revue française de science politique, vol. 22, n. ${ }^{\circ}$ 5, pp. 1074-1110.

ConTe Bernard

1997, «L'aide de l'Union européenne dans le domaine de l'intégration régionale: l'exemple de l'Afrique de l'Ouest», Cahiers du GEMDEV (Paris), n. ${ }^{\circ}$ 25, juin, pp. 155-165.

D'Almeida-Topor Hélène et Lakroum Monique, 1994, L'Europe et l'Afrique. Un siècle d'échanges économiques. Paris, Armand Colin, 235p.

DREUX-BRÉZÉ Joachim de

1968, Le problème du regroupement en Afrique équatoriale. $\mathrm{Du}$ régime colonial à l'Union Douanière et Economique de l'Afrique Centrale. Paris, LGDJ, 211p.

De França Lima Ivaldo Marciano, voir Lima Ivaldo Marciano de França, infra

Doutey Cathy et Gauthé Thierry

2016, «Cartographie des investissements chinois dans le monde» du 10 octobre. Disponible sur <http://www.courrierinternational.com/grand-format/infographie-cartographie-des-investissements-chinois-dans-le-monde>, consulté le 5 janvier 2017.

GauTron Jean-Claude

1977, En attendant Godot ou l'intégration régionale en Afrique noire dans l'évolution récente du pouvoir en Afrique. Bordeaux, CEAN - Centre d'Étude d'Afrique Noire.

Gonidec Pierre-François

1987, Les organisations internationales africaines. Paris, L'Harmattan, 303p. 
Hamilton A. J.

1981, «Les moyens de fonctionnement des organisations interétatiques africaines», Penant n. ${ }^{\circ}$ 774, p. 23.

Kodjo Eden e Chanaiwa David

2010, «Pan-Africanismo e libertação» in Ali Mazrui e Christophe Wondji (eds.), História geral da África, vIII: África desde 1935. Brasília, UNESCO - Representação no Brasil; Ministério da Educação e Universidade de São Carlos, pp. 897-924.

KouAssi E. K.

1987, Organisations internationales africaines. Paris, Berger-Levrault, 485p.

LABAYLE Henri

1983, «La coopération économique régionale en Afrique australe: le temps de la réflexion», Année africaine, pp. 170-211.

1981, «La SADCC ou les difficultés d'une coopération économique régionale en Afrique australe», Année africaine, pp. 254-289.

Lima Ivaldo Marciano de França

2011, «Todos os Negros são Africanos? O Panafricanismo e suas ressonâncias no Brasil contemporâneo», São Paulo, Anais do XXVI Simpósio Nacional de História - ANPUH.

LungYeki MaKiADi Simão, voir MAKIADI Simão Lungyeki, infra

MAKIADI Simão Lungyeki

2016, Do Panafricanismo à União Africana. Seminário de História. Luanda, Departamento de História, Faculdade de Ciências Sociais da Universidade Agostinho Neto (UAN).

2011, L'intégration régionale en Afrique australe: de la Ligne de Front à la SADC. Villeneuve d'Ascq, ANRT, Thèse de Doctorat en Histoire contemporaine, 969p.

MaNDAZA Ibbo 1994, Southern Africa in the 1990s: Problems and prospects of regional cooperation. Harare, SAPES Books.

MaURY Frédéric

2016, "Sylvie Guillaumont: "Le CFA n'est pas si mauvais"», du 2 décembre, sur <http://www.jeuneafrique.com/379249/ economie/sylviane-guillaumont-cfa-nestmauvais/?utm_ source $=$ Newsletter_JA_Eco\&utm_medium $=$ Email \&utm_ campaign=Newsletter_JA_Eco_02_12_16>, lu le 3 décembre 2016.

Nkrumah Kwame

1964, L'Afrique doit s'unir. Traduit de l'anglais par L. Patterson. Paris, Payot. 
OBEnga Théophile

2012, État fédéral d'Afrique noire: la seule issue. Paris, L'Harmattan, 74p.

O. Da Silva Alvaro, voir Silva Álvaro O. da, infra OUA-CM

1968, Groupements régionaux, 11e session ordinaire. Alger, 21 p. CM/231 (Part 5).

1968, Groupements régionaux et intégration des marchés, 11e session ordinaire. Alger, 2 p. cM/Res. 159 (XI).

PAIRAULT Thierry

2016, «À propos de l'investissement chinois en Afrique», du 25 février, disponible sur < https://www.pairault.fr/sinaf/index.php/ statistiques/786-a-propos-de-l-investissement-chinois-en-afrique $>$, consulté le 5 janvier 2017.

PARKoUdA Martin

1980, La contribution des organisations internationales africaines au développement politique du continent africain. Thèse de Droit, Université de Poitiers, 209p.

ReIS DE Almeida Érica, voir Almeida Érica Reis de, supra

RoONEy David

1990, Nkrumah. L'homme qui croyait à l'Afrique. Paris, Éditions Jeune Afrique, 207p.

S.K.B. Asante e Chanaiwa David, voir Asante S.K.B. e Chanaiwa David, supra

Silva Álvaro O. da

1991, Afrique australe à la recherche d'une identité. Paris, L'Harmattan, 1991, 185p.

SUAREZ Alfredo 2009, Intégration régionale. Évolution d'un concept. Paris, Hachette, $158 \mathrm{p}$.

\section{VEREz Jean-Claude} 1998, «Le rôle du secteur informel dans un contexte de régionalisation», Revue du Tiers-Monde, vol. 39, n. ${ }^{\circ}$ 155, pp. 581-596. 
Título: A integração regional em África: Um processo antigo..., para que resultados?

Resumo: Este artigo faz uma análise critíca da história e do estado actual da integração económica e política do continente africano. Constata que, apesar dos esforços consideráveis desenvolvidos a este respeito, a integração regional não parece ter produzido ainda os resultados esperados. Começa por identificar os desafios que os programas de integração regional em África enfrentam, desde o seu surgimento com o ideal panafricano até ao presente, bem como os obstáculos que África deve superar para atingir os seus objectivos previamente traçados pelas suas várias Comunidades económicas regionais (CER). Como conclusão, o artigo apresenta algumas perspectivas de esperança, uma vez que África não está condenada ao desespero tal como os outros continentes, nomeadamente do Terceiro Mundo, um termo em via de desaparecimento do vocabulário das relações internacionais desde o fim da Guerra Fria (1947-1989).

Palavras-chave: África, cooperação, desenvolvimento, integração reginal, organização internacional regional, panafricanismo, regionalismo, unidade africana.

\section{Simão Lungyeki Makiadi}

Est docteur en Histoire contemporaine de l'Université de Paris I - Sorbonne (France). Il est enseignant d'Histoire de l'Afrique au Département d'Histoire (DH) de la Faculté des Sciences Sociales (FCS) de l'Université Agostinho Neto (UAN). Il est auteur de l'ouvrage publié sous le titre de L’intégration régionale en Afrique australe: De la Ligne de Front à la $S A D C$ (2011). Il est aussi auteur des travaux universitaires non publiés: Les Organisations internationales régionales en Afrique australe: Le cas de la SADCC (1995); L’Angola, 1961-1974: La guerre d'indépendance (1994); Le traité franco-allemand (1993); et Les sophistes grecs (1992). Au cours de ses activités professionnelles antérieures et de sa carrière récente d'enseignant ainsi que tout au long de sa formation universitaire, il a participé à diverses activités académiques (conférences, tables rondes, séminaires), où il a été conférencier des thèmes ci-après: Le panafricaniste Kwame Nkrumah et la construction de l'unité africaine (Luanda, 2016); L'Afrique australe: De la discorde à la concorde, le parcours politique d'une intégration régionale en construction (EHESS, Paris, 2003); L'Afrique australe: de la SADCC à la SADC (CRA, Paris, 1997); Les conflits armés en Afrique (Poitiers, 1994); et La politique culturelle de la RPA (Berlin, 1987). Il a était l'un des responsables du Service des Relations internationales du Secrétariat d'État angolais à la Culture (1980-1990), ancien membre fondateur de l'Union Nationale des Artistes Plasticiens (UNAP), ancien membre et secrétaire des Comités nationaux de l' International Council of Museums (ICOM, Conseil international des musées) et de l' International Council on Monuments and Sites (Icomos, Conseil international des monuments et des sites).

[e-mail: simao.lungyeki@sfr.fr] 